\title{
Electron Holography Unveiled Formation Process of Extraterrestrial Magnetite
}

\author{
Yuki Kimura $^{1}$, Kazuo Yamamoto ${ }^{2}$ and Takeshi Sato ${ }^{3}$ \\ 1. Institute of low temperature science, Hokkaido University, Sapporo, Japan. \\ 2. Nanostructures Research Laboratory, Japan Fine Ceramics Center, Nagoya, Japan. \\ 3. Hitachi High-Technologies Corporation, Hitachinaka, Japan.
}

Primitive minerals formed in the solar nebula coagulated into asteroids together with some water ice at the beginning of our Solar system. Interior temperature of an asteroid elevates above the melting point of water ice as a result of mainly the decay heat of radiogenic aluminium-26 [1] and possibly highly energetic impacts [2]. Then, primitive minerals were altered by aqueous processes. The degree of alteration depends on the size of asteroid and the time of asteroidal formation. Therefore, final products in a meteorite allow us estimation of the history of the early solar system. Magnetite particles founding in meteorites has been known as resultants of aqueous alteration in asteroids.

Tagish Lake meteorite, which originated from a D-type asteroid located near the orbit of Jupiter [3], has a lots of magnetite particles with diameters of $110-680 \mathrm{~nm}$ in a significant form. Thousands to ten thousands of the magnetite particles have been assembled in three-dimensionally as colloidal crystals with spherical shape [4]. Whereas framboidal magnetite, which is aggregate of magnetite particles, has been found in several carbonaceous meteorites, Tagish Lake meteorite is only a case for discovery of colloidal crystal. Colloidal crystal is only able to form by an appropriate repulsive force between the particles in a solvent with limited space. If the magnetite particles were typical magnets, they would have readily coagulated as a result of the strong attractive magnetic interactions and they would not have produced a well-ordered structure [5]. Therefore, it is very surprising that how they arranged in three dimensionally and preserved the structure for 4.6 billion years.

The residual magnetization of rocks and minerals is very sensitive to the chronology of their formation and to the temperatures that they have experienced [6]. By means of electron holography, we applied the paleomagnetic method at a nanometer scale (resolution $\sim 6 \mathrm{~nm}$ ) to magnetite particles isolated from colloidal crystals from the Tagish Lake meteorite and we inferred the occurrence of water in the parent body and formation process of magnetite particles.

Electron holography permits the visualization of nanometer-scale magnetic properties and internal electric potentials of samples by recording electron-interference patterns (holograms). We used a transmission electron microscope (Hitachi HF3300-EH) in Japan Fine Ceramics Center. The TEM has been designed specifically for electron holography and has additional sample stage kept in a magnetic field-free environment. We selected colloidal crystals of magnetite together with two, three, four or six particles. The samples were not exposed to any artificial magnetic field at any stages during the experiments.

The magnetite particles extracted from a colloidal crystal have a vortex structure with no external magnetic field, unlike common ferromagnetic materials. There is no report of any natural occurrence of such a vortex structure, although a theoretical prediction based on a three-dimensional micromagnetic simulation has been made of the existence of a uniformly magnetized single-domain vortex state at about $100 \mathrm{~nm}$ [7]. We summarized their circulating magnetic property permits the formation and 
preservation of the three-dimensional colloidal crystals of magnetite in a water droplet, which is required for arrangement by an appropriate repulsive force between the particles. The distribution of colloidal crystals in a small area and its shape suggest that many isolated liquid droplets were distributed in the asteroid and that large-scale assemblies of fluids were absent at least during the nucleation of the particles and the formation of the colloidal crystals. The uniformity of the size distribution and the similar morphology of the framboidal magnetite particles in the colloidal crystals suggest they were formed through homogeneous nucleation from a highly supersaturated solution in a single event. The solution can be formed by following scenario: Calcium and magnesium in a solution initially precipitate as a carbonate during changes in the aqueous environment of the asteroid. The resulting solution is then relatively enriched in iron ions, which was able to spatter by shaking of parent asteroid due to collision of other asteroid and flowed in a cavity of interior the parent asteroid.

Recently, we started in-situ heating experiments to obtain more direct information about the formation history including the temperature and its period of the magnetite formation in a transmission electron microscope. In the presentation, we will show the primitive results.

\section{References:}

[1] M. Endress, et al., Nature 379 (1996), p. 701.

[2] A. F. Rubin, Geochim. Cosmochim. Acta 90 (2012), p. 181.

[3] T. Hiroi, et al., Science 293 (2001), p. 2234.

[4] J. Nozawa et al., J. Am. Chem. Soc. 133 (2011), p. 8782.

[5] A. P. Philipse, D. Maas, Langmuir 18 (2002) p. 9977.

[6] B. P. Weiss et al., Science 290 (2000), p. 791.

[7] M. Winklhofer et al., J. Geophys. Res. 102 (1997) p. 22695.

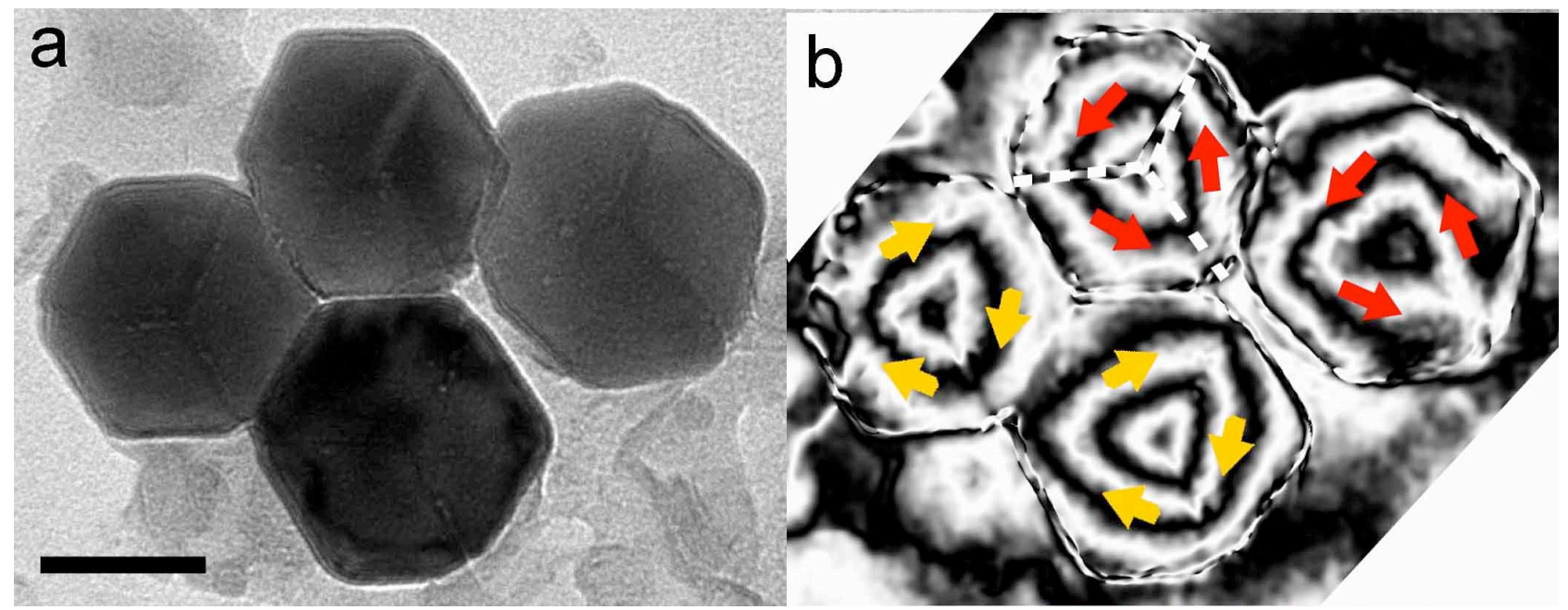

Figure 1. TEM images of magnetite particles in a portion of a colloidal crystal recorded by Hitachi, HF3300-EH (300 kV acceleration voltage). (a) Bright-field image. The scale bar is $100 \mathrm{~nm}$. (b) Phase image of (a) showing that the particles have a concentric circular magnetic field and a vortex structure. Arrows indicate the directions of the magnetization vectors in each magnetite particle. (Reproduced from Nature Communications with permission, doi: $10.1038 /$ ncomms3649) 